\title{
LES COLÉOPTÈRES PARASITES ACCIDENTELS DE L'HOMME ET DES ANIMAUX DOMESTIQUES. (Quatrième note)
}

\section{Par Jean THÉODORIDĖS}

Dans trois notes précédentes parues ici-même (Théodoridès, 1948, $1949 a, 1949 b$ ), j'avais tenté de réunir tout ce que l'on connaissait sur l'importance médicale et vétérinaire des Coléoptères, principalement en tant que parasites accidentels ou mieux pseudoparasites.

Mais on ne peut plus, à l'heure actuelle, prétendre avoir eu connaissance de tout ce qui a paru, même sur un sujet restreint comme celui qui nous intéresse ici et sur lequel j'ai trouvé depuis plusieurs références d'observations récentes; de plus, M. le Prof. Monod a bien voulu me communiquer un cas personnel et original, aussi ai-je cru bon de compléter mes trois notes précédentes par cette quatrième.

\section{1. - Coléoptères parasites accidentels de l'homme.}

L'ordre adopté ici est celui de ma première note (1948).

\section{a. - Tube digestif.}

Deux travaux importants rapportent des cas de scarabiase dans l'Inde ; Strickland et Roy (1939) ont fait une mise au point sur les principaux cas signalés dans ce pays et Ceylan, tout en ajoutant 15 autres cas où les Coléoptères incriminés furent communiqués à des médecins. La plupart appartenaient au genre Onthophagus, quelques-uns au genre Caccobius (Scarabæidæ) et un exemplaire au genre Saprinus (Histeridæ). L'infestation avait lieu surtout chez des enfants.

Chatterjee (1943) signale la présence d'un exemplaire de Scarabéide du genre Caccobius dans les selles d'un enfant de un an et demi, qui avait eu une fièvre continuelle pendant 15 jours et qui avait les voies urinaires infectées par Bacillus coli ; deux exemplaires identiques avaient été évacués «per anum » quelques jours auparavant, mais s'étaient envolés avant d'avoir pu être recueillis. Après l'expulsion des Coléoptères, la température baissa et l'auteur hindou suppose que la pyrexie était causée par la présence de ceuxci dans l'intestin.

Ann. de Parasttologre, T. XXV, $\mathrm{N}^{\circ \mathrm{n}} 1-2$. -1950 , p. 69-76. 
En Afrique du Sud, un cas de scarabiase à Onthophagus sp. chez un enfant indigène a été signalé par de Meillon (1940) et un cas de canthariase a été rapporté dans un compte rendu annuel anonyme (1941), qui mentionne la présence d'une larve de Nitidulide indéterminée, obtenue par sonde stomacale chez un Européen qui prétendait vomir du sang et des «vers » depuis trois mois; avec le Coléoptère, se trouvaient 14 larves de Fannia scalaris F. et 4 de Eristaloides sp. (Diptères).

\section{b. - Voies auditives.}

Le travail de Lopez-Neyra et Roselly (1935), mentionné in NeveuLemaire dans ma note de 1948, décrit le cas d'une personne qui dormait sur de la paille dans un champ près de Grenade (Espagne) et qui eut pendant la nuit l'oreille accidentée (cf. Théodoridès, 1948) par la pénétration du Carabique Acinopus picipes Ol. (1).

Voici, en résumé, l'essentiel de ce cas : il s'agit de Silverio P. F..., célibataire de 24 ans, sans antécédents importants. Le 6 juin 1934, alors qu'il dormait en plein air, comme mentionné ci-dessus, il fut réveillé brusquement par un violent bruit dans l'oreille gauche, qu'il compara à celui fait par une locomotive ; il s'ensuivit une très forte douleur, qui de l'oreille s'étendit à la région temporale et au maxillaire supérieur; le frère du patient pensant qu'un animal avait pénétré dans l'oreille, essaya, mais en vain, de le retirer avec un brin de paille.

La douleur persistant tout le jour, et la nuit suivante, le patient s'en fut consulter le médecin de son village qui ne pratiqua pas d'otoscopie et ne put que constater dans l'oreille du sang et des matières purulentes, concluant qu'il s'agissait d'une affection grippale et prescrivant un calmant local.

Deux jours après, la suppuration avait augmenté, et le malade se sentait fiévreux, sans toutefois vérifier sa température; à ceci s'ajoutaient des nausées et vomissements.

C'est alors que le patient fut amené au $\mathrm{D}^{x}$ Roselly, oto-rhino-laryngologiste, qui procéda à un examen détaillé : la bouche et le pharynx étaient normaux, le nez présentait un léger coryza avec sécrétion muco-purulente. L'oreille droite était normale, alors que la gauche montrait une grande infiltration du conduit auditif et une suppuration fétide; à la palpation, la douleur put être localisée au-dessus de la zone cartilagineuse du conduit auditif, au-dessus de la mastoöde et à la zone antrale.

Un lavage fut pratiqué avec la seringue servant pour extraire les corps

(1) Je remercie ici M. le Prof. Lopez-Neyra, de l'Université de Grenade (Instituto Nacional de Parasitologia), qui a bien voulu m'envoyer dans un délai très bref ses travaux cités dans cette note. 
étrangers, ainsi que l'otoscopie : le conduit était rempli dans toute sa largeur par un corps étranger brillant, strié et de couleur noire.

Il y avait de plus un nystagmus spontané d'origine labyrinthique.

Vu l'état général alarmant du malade (fièvre, affaiblissement, céphalée, etc...), l'extraction du corps étranger fut immédiatement pratiquée : le conduit auditif fut anesthésié localement avec du liquide de Bonain et le corps extrait à l'aide d'une pince fine ; l'on vit alors qu'il s'agissait de la partie abdominale d'un Coléoptère.

L'otoscopie fut répétée et le conduit maintenant libre permit de voir le tympan perforé en ses deux quadrants antérieurs, laissant apparaitre une bonne partie du manche du marteau; à travers la perforation se voyait un corps étranger noirâtre qui devait être l'autre fragment du Coléoptère.

L'anesthésie locale fut alors répétée et le second fragment extrait de la même façon que le premier s'avéra être en effet la tête et le prothorax du Coléoptère.

Une troisième et dernière otoscopie permit de constater que la caisse tympanique était désormais vide, bien que présentant des parois infiltrées et suppurantes.

Après un lavage à l'eau oxygénée, le malade fut reconduit à son domicile et on lui prescrivit un lavage de l'oreille, toutes les 6 heures, avec un mélange de 1 partie de liqueur de Labarraque et de 5 parties d'eau bouillie tiède, ainsi que l'instillation de 7 à 8 gouttes d'une solution de protargol à $3 \%$ entre les lavages et l'inhalation d'alcool menthé à $1 \%$, pour le nez, plusieurs fois par jour.

Le 26 juillet, le malade était complètement rétabli.

Les auteurs estiment que sans l'extraction du Coléoptère, ce cas aurait pu être fatal par suite de complications intracraniennes et rappellent brièvement les autres cas signalés, dans la littérature médicale, de pénétration accidentelle d'insectes dans l'oreille.

Le Carabique incriminé ici put être déterminé par le Prof. D.-C. Bolivar comme étant Acinopus picipes Ol., insecte de dimension relativement grande $(17 \mathrm{~mm}$. de longueur). Les auteurs pensent que ce Coléoptère en quête d'un gîte avait pénétré par erreur dans l'oreille du sujet et, s'étant heurté au tympan, l'avait percé avec ses mandibules acérées, en cherchant à aller plus loin ; ne pouvant ensuite ni avancer, ni sortir, il s'enfonçait chaque fois plus fort dans l'oreille, causant chez le patient les violentes douleurs mentionnées plus haut.

La pénétration accidentelle de Coléoptères dans l'oreille peut donc facilement survenir à la faveur de séjours prolongés dans la nature, surtout la nuit.

Les lignes suivantes rapportent un cas de pénétration, heureusement sans gravité : 
M. le Prof. Th. Monod, Directeur de l'Institut français d'Afrique Noire, a bien voulu me communiquer un cas personnel et original de pénétration accidentelle d'un coléoptère dans l'oreille qu'il a raconté de façon si pittoresque et imagée dans son livre «Méharées » (1937) (2) ; il s'agit non pas d'un Carabique, mais de Mesostena angustata Fabr. (Tenebrionidæ), espèce qui doit être commune dans le Sahara occidental. Heureusement pour l'auteur de "Méharées », l'insecte put être retiré à temps, car le Coléoptère en s'agitant aurait pu percer le tympan et envahir la caisse tympanique comme dans le cas espagnol cité ci-dessus. D'ailleurs, le Prof. Etienne, de la Faculté de Médecine de Montpellier, écrivit à ce sujet au Prof. Monod (3) :

« Dans votre livre..., vous narrez les exploits d'un coléoptère qui avait confondu votre conduit auditif externe avec quelque galerie accueillante, et terminez cette petite narration par le mot " ridicule ». Vous me permettrez de dire que ce n'est pas ridicule du tout, c'est très désagréable, même à certains égards dangereux. Si par hasard un nouvel incident vous arrivait, ni sable ni eau, mettez de l'huile dans le conduit auditif externe. G'est la mort du coléoptère par asphyxie. Son extraction doit être ensuite faite de préférence avec un jet d'eau de très faible diamètre projeté vers le haut du conduit auditif externe.»

Enfin, à propos des cas de pénétration accidentelle de Coléoptères dans l'oreille, il est intéressant de noter la chose suivante : dans ma note de $1949 b$, je signalais (p. 119, note 1) le fait rapporté par P. de Miré, qu'un Bostrychide indéterminé du Sahara occidental était redouté des indigènes du fait de sa pénétration dans l'oreille externe des dromadaires.

Or, dans un récent travail (Español, 1947), sur des Coléoptères du Sahara espagnol, on peut lire (loc. cit., p. 353), à propos du Bostrychide Sinoxylon senegalense Karsch : " En nuestras posesiones saharianas este Sinoxylon es bien conocido por la población indi-

(2) « Au puits de Touijmert, incident grotesque. Un coléoptère noir, sorte de carabe, s'est introduit dans mon oreille gauche, convaincu d'avoir trouvé un petit coin tranquille pour passer la nuit. Je ne l'entendais pas de la sorte - si je puis dire - et ai protesté sans succès. Puis, connme l'hôte inconnu grouillait toujours, et qu'un carabe dispose de 6 pattes, 2 antennes, 2 mandibules, j'ai convoqué la lampe à carbure et Sidi. Ce dernier, ne voyant rien, propose de me remplir l'oreille de sable, pour décourager le locataire. Je suggère l'eau plutôt que le sable, ce qui fut fait. Mais le carabe grincheux en conçut une colère si terrible qu'il se mit à taper des pieds et à se livrer à une série d'acrobaties douloureuses (pour le propriétaire de l'appartement) avant de se résigner à faire machine arrière. Aussitôt vu, il fut expulsé. Ridicule. » (Méharées, p. 186).

(3) Je remercie ici M. le Prof. Monod qui a bien voulu me communiquer la lettre que lui a adressée le Prof. Etienne et qui m'a si aimablemert autorisé à publier ce cas personnel et original. 
gena, y muy temido... por la creencia, bastante arraigada, de que es capaz de penetrar en la cabeza, a través del oido, y ocasionar la muerte del individuo atacado. »

Ce Coléoptère est peut-être en effet responsable d'accidents de l'oreille chez les indigènes, mais il est très difficile d'affirmer ceci en toute certitude ; ce qui est en tout cas curieux, c'est de voir qu'à deux endroits différents du Sahara, la même croyance persiste chez les indigènes qui donnent au Bostrychide incriminé le même nom : « sousse » au Sahara français, "susa au Sahara espagnol.

\section{c. - Voies urinaires.}

Jack (1940) mentionne la présence d'une larve de Coléoptère indéterminée dans les voies urinaires d'un enfant européen, en Rhodésie du Sud. A propos d'un cas de myiase génito-urinaire à Wohlfahrtia magnifica (Schin.), Lopez-Neyra et Estévez (1949) viennent de faire une révision de tous les cas connus de ces myiases ( $\&$ uretromiasis ») et pensent que, dans certains de ceux-ci, il pourrait s'agir en fait de larves de Coléoptères (canthariases).

\section{d. - Gas d'ectoparasitisme.}

Van Dyke (1932) mentionne incidemment, à propos de la biologie des Staphylinides nidicoles du genre Quedius nidicoles et pholéophiles, que $Q$. pedicularius (4) se rencontre dans les cheveux des Esquimaux de l'Alaska. Chez un paysan de Saône-et-Loire, Harant (1937) (5 bis) a signalé un intense prurit du cuir chevelu, persistant depuis plusieurs jours et dû à la présence sur la tête du patient d'un nombre important de larves triongulins (Meloidæ) indéterminées; ce pseudoparasitisme a cédé à un savonnage important des cheveux. Le paysan a déclaré s'être allongé et avoir dormi pendant deux heures dans son potager, ce qui explique l'étiologie de cette curieuse infestation. Ce seraient là les deux seuls cas d'ectoparasitisme accidentel de Coléoptères chez l'homme, alors que dans d'autres ordres (Collemboles par exemple), des espèces vivant en général libres ont été rencontrées accidentellement chez l'homme, en particulier dans les cheveux (5).

(4) Cette espèce ne figure pas dans le «Coleopterorum Catalogus » Junk, et d'après M. J. Jarrige, attaché au Laboratoire d'Entomologie du Muséum, il s'agirait de $Q$. pediculus Nordm. (ce nom d'espèce n'ayant d'ailleurs rien à voir avec la localisation accidentelle de ce Coléoptère dans des cheveux humains).

(5) J'ai l'intention de rédiger, dans un avenir proche, une mise au point sur cette question, pour les «Annales de Parasitologie », en collaboration avec M. Delamare Deboutteville, spécialiste de ce groupe.

(5 bis) Je remercie ici M. le Prof. Harant pour cet intéressant renseignement et pour le chaleureux accueil qu'il m'a réservé dans son laboratoire, lors de mon séjour à Montpellier. 
Pour terminer cette partie consacrée aux cas de parasitisme accidentel chez l'homme, je propose qu'à l'avenir on adopte le terme de canthariase pour désigner uniquement l'infestation du tube digestif par des larves de Coléoptères (6) et celui de scarabiase pour les cas d'infestation par des adultes.

\begin{tabular}{|c|c|c|c|c|}
\hline \multicolumn{2}{|c|}{ COLÉ́OPTÈRES } & \multirow{2}{*}{$\begin{array}{l}\text { IIIEU D'TNFES- } \\
\text { TATION CHEZ } \\
\text { L'HOMME }\end{array}$} & \multirow{2}{*}{ LOCALITÉ } & \multirow{2}{*}{ AUTEURS } \\
\hline $\begin{array}{c}\text { Familles } \\
(\mathrm{L}): \text { larve }\end{array}$ & $\begin{array}{l}\text { Espèces } \\
\text { (A) : adulte }\end{array}$ & & & \\
\hline Staphylinide. & $\begin{array}{l}\text { Quedius pedicula- } \\
\text { rius (sic) (A?) }\end{array}$ & Cheveux & Alaska & Van Dyke (1932) \\
\hline Histerida $\quad \ldots$ & Saprinus sp. (A) & Intestin & Inde & $\begin{array}{l}\text { Strickland } \\
\text { et Roy (1939) }\end{array}$ \\
\hline \multirow[t]{2}{*}{ Scarabæidx . } & $\begin{array}{l}\text { Onthophagus sp. } \\
\text { (A) }\end{array}$ & Intestin & Inde & $\begin{array}{l}\text { Striekland } \\
\text { et Roy (1939) }\end{array}$ \\
\hline & $\begin{array}{l}\text { Caccobius sp. } \\
\text { (A) }\end{array}$ & $\begin{array}{l}\text { Intestin } \\
\text { Intestin }\end{array}$ & $\begin{array}{c}\text { Inde } \\
\text { Inde (Assam) }\end{array}$ & $\begin{array}{c}\text { Strickland } \\
\text { et Roy (1939) } \\
\text { Chatterjee (1943) }\end{array}$ \\
\hline $\begin{array}{ll}\text { Nitidulida } & . .\end{array}$ & $\begin{array}{c}\text { Larve } \\
\text { indéterminée } \quad(\mathrm{L})\end{array}$ & Estomac & $\begin{array}{l}\text { Afrique } \\
\text { du Sud }\end{array}$ & $\begin{array}{c}\text { Auteur anonyme } \\
(1941)\end{array}$ \\
\hline Ptinida $\ldots .$. & $\begin{array}{l}\text { Ptinus tectus } \\
\text { Boield (L) }\end{array}$ & Intestin & Angleterre & Sharpe (1947) \\
\hline Meloida .. & $\begin{array}{l}\text { Larves triongulins } \\
\text { indéterminées }\end{array}$ & Cheveux & France & Harant (1937) \\
\hline \multirow[t]{3}{*}{ Tenebrionidæ. } & $\begin{array}{c}\text { Blaps mortisaga } \\
\text { L. (L) }\end{array}$ & Intestin & Angleterre & Cobbold (1877) \\
\hline & $\begin{array}{l}\text { Tenebrio molitor } \\
\text { L. (A?) (ou autre } \\
\text { Ténébrionide) }\end{array}$ & Conjonetive & $\begin{array}{l}\text { Tchéco- } \\
\text { slovaquie }\end{array}$ & Koldovsky (1930) \\
\hline & $\begin{array}{l}\text { Mesostena } \\
\text { angustata Fabr. } \\
\text { (A) }\end{array}$ & $\begin{array}{l}\text { Oreille (péné- } \\
\text { tration acci- } \\
\text { dentelle sans } \\
\text { complication) }\end{array}$ & $\begin{array}{l}\text { Sahara } \\
\text { Occidental }\end{array}$ & Monod (1937) \\
\hline \multicolumn{2}{|c|}{ Larve indéterminée (L) } & $\begin{array}{l}\text { Voies } \\
\text { urinaires }\end{array}$ & $\begin{array}{l}\text { Rhodésie } \\
\text { du Sud }\end{array}$ & Jack (1940) \\
\hline
\end{tabular}

(6) Elargissant ainsi la définition donnée par Hope, qui englobait également sous le terme de "canthariasis 》 les cas d'infestation par des nymphes ou même des adultes de Coléoptères; en pratique, les nymphes ne sont jamais 
En groupant les quelques cas signalés dans ma note complémentaire $(1949 a)(7)$ et dans celle-ci, le tableau ci-dessus complète celui de la première note (1948).

\section{2. - Coléoptères parasites accidentels d'animaux domestiques,}

Depuis ma note précédente $(1949 b)$, j'ai trouvé quelques références additionnelles concernant les Coléoptères qui peuvent s'attaquer accidentellement aux oiseaux des basses-cours : la larve de Dermestes lardarius L. peut pénétrer dans des œufs de canes (8) lorsque la coquille de ceux-ci se brise au moment de l'éclosion et s'attaque aux jeunes canetons ; l'on a remarque jusqu'à 6 et même 8 larves dans un seul œuf (observation anonyme en Allemagne, 1917); une autre note (anonyme également, 1919) rappelle l'observation précédente et rapporte le cas d'un poulailler du Mecklembourg (Allemagne) dans lequel une centaine de canetons et de poussins juste éclos des œufs furent attaqués et saignés à mort par les larves de $D$. lardarius, les poules couveuses étant aussi mordues au sang. Le poulailler fut nettoyé avec une solution de lysol et avec de l'eau de soude (sodawasser). En nettoyant, on découvrit que les Dermestes provenait d'un pigeonnier (qui se trouvait au-dessus du poulailler), ceci s'expliquant fort bien par le fait que ces Coléoptères à l'état larvaire attaquent les jeunes pigeons au nid (cf. note de $1949 \mathrm{~b}$ ). Gibson (1930) et Bunyea et Wehr (1941) ont également rappelé ces cas fort curieux où des Coléoptères nécrophages et saprophages s'attaquent à des oiseaux domestiques vivants, dans les basses-cours (9). Enfin, Cobbold (1879) a mentionné la présence accidentelle de larves du Ténébrionide Blaps mortisaga L. (rencontré aussi chez

rencontrées, et le terme commode de scarabiase (« scarabiasis 》 des auteurs de langue anglaise) a été adopté à l'unanimité par les auteurs (surtout aux lndes) pour désigner les cas d'infestation par des adultes.

(7) Ne seront pas mentionnés ici les pseudo-parasites signalés par Hope (et énumérés dans ma note $1949 a$ ) comme étant des cas trop anciens, d'où sujets à caution.

(8) Dans une de mes précédentes notes $(1949 \mathrm{~b})$, je signalais que Necrophorus vestigator Hersch. (Silphida) pouvait attaquer les jeunes pigeons; Donisthorpe (Entom. Mon. Mag., 66, 1930, p. 277) a signalé la présence de Necrophorus humator Goeze et $\hat{N}$. mortuorum $\mathrm{F}$. (= vespilloides Herbst) à l'intérieur d'un œuf de faisan en état de décomposition, dans la Forêt de Windsor (Angleterre) ; l'auteur anglais suppose que ceux-ci provenaient d'œufs (ou de jeunes larves) déposés là (vraisemblablement par une fissure de la coquille), car on peut difficilement expliquer autrement la présence de ces deux Nécrophores adultes.

Ce cas très curieux n'est pas sans analogie avec celui des Dermestes mentionnés ci-dessus.

(9) Marotel les cite également dans sa récente \& Parasitologie Vétérinaire 》 (1 vol. 652 pp. Encyclopédie Cadillac, Baillière édit., Paris, 1949), mais il est malheureusement très regrettable, dans un ouvrage pédagogique, de voir parler de "tenebrio» (sic), de «silpha» (sic) et d'y lire que la larve de Dermestes lardarius est \& chevelue » (sic)! 
l'homme dans des cas tout à fait exceptionnels; cf. Théodoridès, 1949 a) chez le cheval. Il m'a malheureusement été impossible de consulter l'original de ce travail introuvable à Paris.

\section{BiBLIOGRAPHIE}

(Certains travaux étrangers n'ont pu être consultés, du fait que les revues où ils ont paru sont introuvables à Paris, mais les Analyses de la Review of Applied Entomology (Sér. B) nous en ont fait connaître l'essentiel.)

(Auteur anonyme). - Die Larve des Speckkäfers (Dermestes lardarius L.) ein Feind des schlüpfenden Geflügels. Deutsch. Landwirtschft. Presse (Berlin), XLIV, 1917, 10, 83-4, 2 fig.

(Auteur anonyme). - Speckkäferlarven (Dermestes lardarius L.) als Schädiger im Geflügelstall. Zeitschft. angew. Entom., V, 1918, 130-132.

(Auteur anonyme). - Entomology. Rept. S. Afr. Inst. Med. Res. (1940), 1941, Johannesburg, 26-29.

Bunyea (H.) et Wehr (E. E.). - Diseases and Parasites of Poultry. Farmer's Bull., U.S. Dept. Agric. 1652, 1941, 82 pp., 34 figs, 13 refs.

Chatterjee (B.). - Pyrexia due to beetle infection. Ind. Med. Gaz., LXXVIII, $1943,96$.

Coввoln (T. S.). - On the occurrence of the larvae of Blaps mortisaga in the horse. The Veter. (Lond.), LII, 1879, 473-76.

1)Yке (E. C. van). - (in Sitzungberichte). Mitt. Deutsch. Ent. Ges., III, 1932, $8,114$.

Español (F.). - Pselafidos, Cleridos y Bostriquidos (Col.) del Sahara Español. Eos, XXIII, 1947, 341-55.

GiBson (A.). - Insect and other external parasites of poultry in Canada. Sci. Agric., XI, 1930, 4, 208-20.

Harant (H.), - Parasitisme accidentel par les larves triongulins. Acad. Sci. Lettr. Montpellier, 1937 (communication orale).

J AGK (R. W.), - in: Rept. Div. Ent. (South Rhodesia) for the year ending 31st Dec. $1939,1940,35 \mathrm{pp}$. (dactylographié).

Lopez-Neyra (C. R.) et Roselly (L. J.). - Un caso de escarabajo en el oido, con destruccion de la membrana del timpano e invasion de la caja timpanica. Act. Med. (Granada), juin 1935, 332-35.

LOPEZ-Neyra (C. R.) et Estévez (M. S.). - Primer caso de uretromiasis por Wohlfahrtia magnifica (Schiner 1862). Rev. Iber. Parasit., IX, 1949, 91108.

Meillon (B. de). - Entomology. Rept. S. Afric. Inst. Med. Res., (1939), 1940, 30-37.

Monod (Th.). - Méharées. 1 vol., 300 pp., édit. « Je sers », Paris, 1937.

STrickland (C.) et Roy (D. N.). - Scarabiasis or the presence of beetles in the intestine. Ind. Med. Gaz., LXXIV, 1939, 416-419.

THÉodoridès (J.). - Les Coléoptères parasites accidentels de l'lomme. Ann. Parasit., XXIII, 1948, 348-363.

- Id. Note complémentaire. Ibid., XXIV, 1949a, 110-115.

-. Les Coléoptères nuisibles aux animaux domestiques. Ibid., XXIV. $1949 b, 116-123$.

Station Expérimentale de Richelieu (Indre-et-Loire) et Laboratoire d'Entomologie Coloniale du Museum de Paris. (Adresse actuelle: Laboratoire Arago, Banyuls-sur-Mer, Pyr.-Or.). 
z 
ANNALES DE PARASITOLOGIEE

T. XXV, ${ }^{\circ s} 1-2,1950$

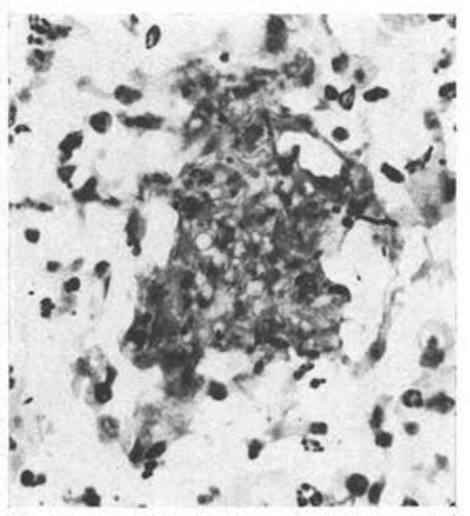

1

2
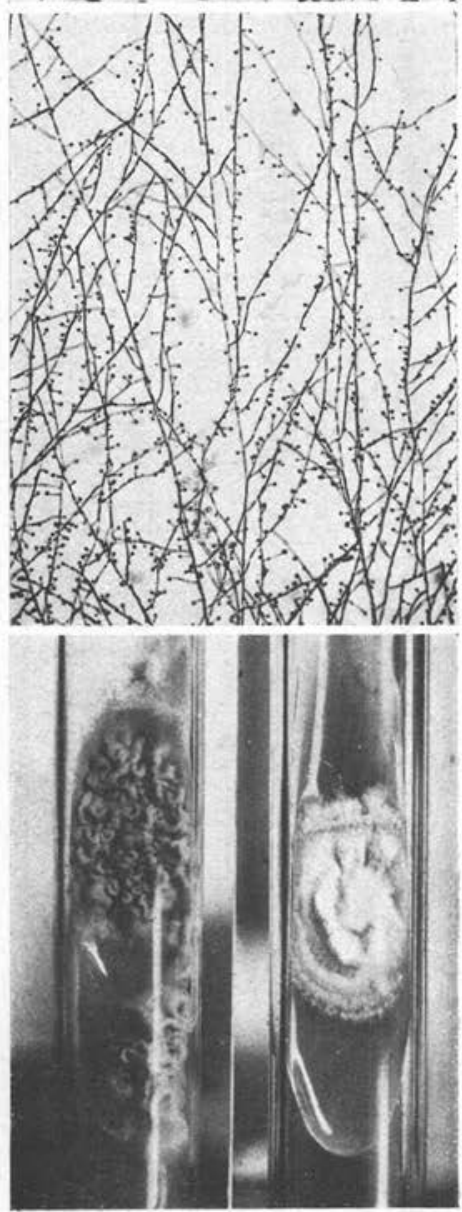

Planche VI

(Mémoire Rodhain el Mattlet)

Fig. 1. - Au centre, un grain jeune ; les taches noires correspondent à la section des filaments mycéliens.

Fig. 2. - Culture-type à sec, de 20 jours.
Fig. 3. - Cultures d'un mois sur gélose de Sabouraud: à gauche, milieu d'épreuve; à droite, milieu de conservation. 